\title{
THERAPEUTIC HYPOTHERMIA GUIDANCE BY CEREBRAL PERFUSION CONTROL BY DOPPLERULTRASOUND AFTER CPR IN CHILDREN
}

\author{
H. Ringe ${ }^{1}$, V. Varnholt ${ }^{1}$, S. Reich ${ }^{1}$, A. Gratopp ${ }^{1}$, A. Rei ${ }^{1}$, A. Kubinski ${ }^{1}$, S. Dähmlow ${ }^{1}$, H. Krude ${ }^{2}$ \\ ${ }^{I}$ Paediatric Intensive Care, ${ }^{2}$ General Paediatrics, Charite Campus Virchow, Berlin, Germany
}

Objectives: Despite hypothermia treatment $(12-24 \mathrm{~h})$ survival after cardiopulmonary arrest (CA) is poor in children compared to adults particularly depending on the number out-of-hospital/in-hospital CPR (7-53\%). We observed cerebral hyperperfusion in children with adverse outcome after 24 h hypothermia post CA during the re-warming by transcranial Doppler-Ultrasound (DU). We interpreted this as reperfusion injury after too early and fast re-warming which might be avoidable by Dopplerultrasound guidance.

Methods: Intention-to-treat protocol: Inclusion criteria: Children with CA in- and out-of-hospital, aged 4 weeks -18 years; lactate $\geq 100 \mathrm{mg} / \mathrm{dl}, \mathrm{CPR} \geq 10 \mathrm{~min}$; given parental consent. Treatment with hypothermia 32$34^{\circ}$ Celsius for 48 hours after CPR and re-warming by $0,5^{\circ} \mathrm{C} / 6 \mathrm{~h}$ under DU steering. If vascular Resistive Index (RI) decreased below 0,6 re-warming was reversed or at RI 0,6-0,65 re-warming was withheld for 24 hours and was re-started after $24 \mathrm{~h}$. No fever is allowed for 7 days following hypothermia. We looked for the outcome (survival, GOS at discharge +6 month) and possible side-effects (bleeding, cardiac arrhythmias, coagulation disturbances, platelet count, infections).

Results: 15 children are included so far. Median for: ROSC 25 (10-120)min, PRISM and PIM Score $>90 \%$, and lactate $159 \mathrm{mg} / \mathrm{dl}$. Median hypothermia-time 147(65-212)hours. In 12 children re-warming was withheld for 24 hours in 8 re-warming was reversed. We saw no severe adverse effects. 12/15 (80\%) patients survived, 9/12(75\%\%) survived without neurological residuals after 6 month.

Conclusions: Our results suggest that hypothermia and re-warming steered by DU might be a safe and lifesaving treatment after CPR. 